\title{
Reddish Diarrhoea Caused by Excessive Ingestion of Watermelon: Mimicker of Bacterial Colitis
}

\section{Kazuto Taniguchi, Toshihiro Nonaka and Kazuyasu Uemichi}

Department of Paediatrics, Tara Town Hospital, Tara, Japan

\section{Correspondence:}

Kazuto Taniguchi

Department of Paediatrics

Tara Town Hospital,

Tara,

Japan

E-mail: tgene1@po.hagakure.ne.jp

DOI: $10.3126 /$ jnps.v41i1.32932

Submitted on: $2020-08-06$

Accepted on: 2021-02-26

Acknowledgements: None

Funding: Nil

Conflict of Interest: None declared

Permission from IRB: Yes

\section{ABSTRACT}

Bloody diarrhoea in children is indicative of serious diseases. Although bloody diarrhoea following bacterial colitis is well known, reddish diarrhoea caused by excessive ingestion of watermelon is unknown. A two year old girl who excessively ingested watermelon presented with repeated reddish diarrhoea. A kit for fecal occult blood testing revealed that the reddish diarrhoea did not contain blood. Reddish diarrhoea caused by excessive ingestion of watermelon can be a mimicker of bacterial colitis. The kit for fecal occult blood testing was useful for differential diagnosis. We should be aware that this pitfall can be hidden in daily nutrition.

Key words: Bacterial colitis; differential diagnosis; reddish diarrhoea; watermelon

To cite this article: Taniguchi $\mathrm{K}$, Nonaka T, Uemichi K. Reddish Diarrhoea Caused by Excessive Ingestion of Watermelon: Mimicker of Bacterial Colitis. J Nepal Paediatr Soc. 2021;41(1):115-8.

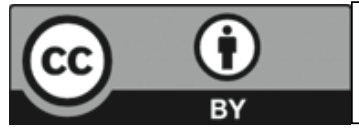

This work is licensed under creative common attribution 3.0 license 


\section{INTRODUCTION}

Bloody diarrhoea in children is indicative of serious diseases. ${ }^{1}$ During summer in particular, bacterial colitis caused by Shiga toxin-producing Escherichia coli (STEC), Salmonella and Campylobacter often presents with severe bloody diarrhoea. ${ }^{2}$ Bacterial colitis can result in haemolytic uremic syndrome (HUS), a life-threatening condition. ${ }^{3,4}$ Watermelon, which contains red flesh with black and white seeds, is popular throughout summer. Although bloody diarrhoea following bacterial colitis is well known, reddish diarrhoea caused by excessive ingestion of watermelon is unknown. Herein we report a two year old girl who was diagnosed with reddish diarrhoea that did not contain blood and instead arose from excessive ingestion of watermelon. A kit for fecal occult blood testing proved useful in the differential diagnosis.

\section{CASE REPORT}

A two year old girl, developmentally normal child, with no significant past history, presented with repeated reddish diarrhoea followed by impaired consciousness. Reddish diarrhoea samples obtained from the patient contained watermelon seeds, suggesting that she excessively ingested watermelon (Figure 1).

On physical examination, she had a pale complexion and impaired consciousness, although her vital signs, including blood pressure and pulse rate, presented no abnormalities. Suspecting gastroenteritis and colitis, she was investigated thoroughly. Blood tests revealed hypoglycemia

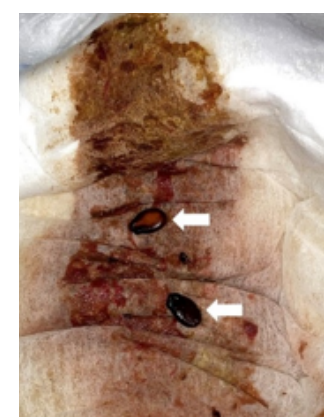

Figure 1. Reddish diarrhoea on a diaper. The reddish diarrhoea contained watermelon seeds (arrowheads)

with serum glucose level of $50 \mathrm{mg} / \mathrm{dL}$, while Creactive protein (CRP) and procalcitonin levels were normal. Other indicators including transaminase, urea nitrogen, electrolytes, and hemoglobin showed no abnormalities. A kit for dual detection of noroviruses and group A rotaviruses in stool samples (IP Line Duo NoroRota; Immuno-Probe, Saitama, Japan) ${ }^{5}$ did not detect any antigens for these viruses. Abdominal Xrays revealed no abnormalities. We employed a kit for fecal occult blood testing (OC-HemocatchS; Eiken Chemistry, Tochigi, Japan), based on immunochromatography (Figure 2), to determine whether the reddish diarrhea contained blood. The kit did not detect any blood for two consecutive days. A culture examination detected no significant bacteria after 4 days.

She received an intravenous drip infusion of physiological saline solution. As soon as the hypoglycemia was corrected by intravenous administration of $50 \%$ glucose solution, she recovered consciousness. Her serum glucose level
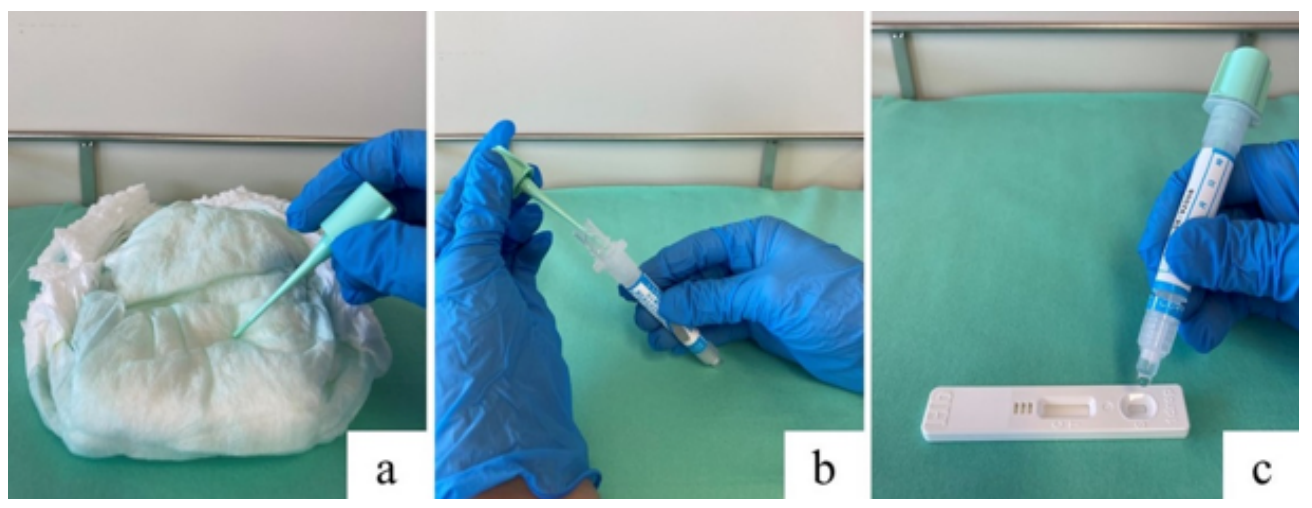

Figure 2. Demonstration of the kit for fecal occult blood testing. (a) A stick equipped in the cap of a tube is dipped in the stool sample. (b) The cap is replaced to immerse the stick in the reagent, and the tube is inverted several times. (c) The extract is dripped onto an immunochromatographic test plate and incubated for five minutes. 
increased to $119 \mathrm{mg} / \mathrm{dL}$ after one hour, and the hypoglycemia did not recur. She also received oral administration of probiotics for 13 days. The reddish appearance of diarrhoea lasted for several days. Diarrhoea remained for approximately 10 days. She recovered without any complications.

\section{DISCUSSION}

We observed two important clinical issues in our patient: reddish diarrhoea caused by excessive ingestion of watermelon can be a mimicker of bacterial colitis, and a kit for fecal occult blood testing can be useful to determine whether reddish diarrhoea contains blood.

Regarding the first issue, reddish diarrhoea obtained from our patient was similar to bloody diarrhoea, the typical symptom of bacterial colitis. Clinical manifestations of bacterial colitis include repeated bloody diarrhoea and abdominal cramping. ${ }^{2,4}$ Severe cases with bacterial colitis present with lethargy following dehydration and hypoglycemia. ${ }^{1,2}$ Moreover, some children with bacterial colitis, especially those infected with STEC, are at risk of developing HUS, which is characterized by microangiopathic haemolysis, platelet destruction, and renal failure. ${ }^{3,4}$ Patients with HUS require intensive care including hemodialysis. ${ }^{4}$ Thus, the differential diagnosis should be performed rapidly.

For the second issue, the differential diagnosis of reddish diarrhoea caused by excessive ingestion of watermelon and bloody diarrhoea resulting from bacterial colitis is difficult because they closely resemble one another in appearance. Because a rapid differential diagnosis is desirable, a culture examination is unsuitable because it requires several days to obtain a definitive result. We utilised a kit for fecal occult blood testing, and found no evidence of blood in the diarrhoea. The improper use of antibiotics can induce not only adverse reactions, but also microbial resistance. Consequently, we were able to avoid administration of antibiotics because no signs indicating bacterial infection were detected in our patient. Measurement of CRP and procalcitonin in blood tests revealed no abnormalities, and culture examination of the reddish diarrhoea detected no significant bacteria. We suggest that the kit for fecal occult blood testing is a useful tool for the differential diagnosis.

Watermelon, botanically considered a fruit, belongs to the family Cucurbitaceae. ${ }^{6,7}$ It contains almost $92 \%$ water, $7.55 \%$ carbohydrates, and $0.4 \%$ dietary fiber. ${ }^{7} 8$ Excessive ingestion of water and fiber can lead to diarrhoea. Most watermelon in Japan contains red flesh with black and white seeds. The main pigment producing the red flesh colour is lycopene, an important natural carotenoid. ${ }^{9}$ Although we did not try to detect lycopene, we assumed that the red pigment in the reddish diarrhoea samples obtained from our patient was derived from lycopene in the watermelon. Watermelon lycopene can prevent cancer, diabetes, hypercholesterolemia, cardiovascular disorders, and macular diseases. ${ }^{7,10}$ Thus, watermelon consumption has escalated owing to its related health benefits. ${ }^{7,10}$ Furthermore, watermelon is the favourite fruit of most children during summer. We should be aware that excessive ingestion of watermelon can be a pitfall in daily nutrition.

\section{CONCLUSIONS}

Reddish diarrhoea caused by excessive ingestion of watermelon can be a mimicker of bacterial colitis, especially in summer. A kit for fecal occult blood testing is useful for the differential diagnosis. We should be aware that this pitfall can be hidden in daily nutrition. Further studies are needed to determine whether excessive ingestion of watermelon can become a major differential diagnosis for reddish diarrhoea in children.

\section{REFERENCES}

1. Murphy MS. Management of bloody diarrhoea in children in primary care. BMJ. 2008;336:1010-5. DOI: 10.1136/ bmj.39542.440417.BE.

2. Shane AL, Mody RK, Crump JA, Tarr PI, Steiner TS, Kotloff K, et al. 2017 Infectious Diseases Society of America clinical practice guidelines for the diagnosis and management of infectious diarrhea. Clin Infect Dis. 2017;65:e4580. DOI: $10.1093 / \mathrm{cid} /$ cix669. 
3. Loconsole D, Giordano M, Centrone F, Accogli M, Casulli D, De Robertis AL, et al. Epidemiology of Shiga toxinproducing Escherichia coli infections in Southern Italy after implementation of symptom-based surveillance of bloody diarrhea in the pediatric population. Int J Environ Res Public Health. 2020;17:5137. DOI: 10.3390/ ijerph17145137.

4. Ylinen E, Salmenlinna S, Halkilahti J, Jahnukainen T, Korhonen L, Virkkala T, et al. Hemolytic uremic syndrome caused by Shiga toxin-producing Escherichia coli in children: incidence, risk factors, and clinical outcome. Pediatr Nephrol. 2020;35:1749-59. DOI: 10.1007/s00467-020-04560-0.

5. Khamrin P, Kumthip K, Thongprachum A, Takanashi S, Okitsu S, Maneekarn N, et al. Evaluation of immunochromatographic test for dual detection of noroviruses and group A rotaviruses in stool samples.Clin Lab . 2018;64:793-6. DOI: 10.7754/Clin.Lab.2017.171201.

6. Edwards AJ, Vinyard BT, Wiley ER, Brown ED, Collins JK, Perkins-Veazie P, et al. Consumption of watermelon juice increases plasma concentrations of lycopene and beta-carotene in humans. J Nutr 2003;133:1043-50. DOI: 10.1093/jn/133.4.1043.

7. Naz A, Butt MS, Sultan MT, Qayyum MMN, Niaz RS. Watermelon lycopene and allied health claims. EXCLI J 2014;13:650-60.

8. Leskovar D, Bang H, Crosby K, Maness N, Franco A, Perkins-Veazie P. Lycopene, carbohydrates, ascorbic acid and yield components of diploid and triploid watermelon cultivars are affected by deficit irrigation. J Hortic Sci Biotechnol. 2004;79:75-81. DOI: 10.1080/14620316.2004.11511739.

9. Wang C, Qiao A, Fang X, Sun L, Gao P, Davis AR, et al. Fine mapping of lycopene content and flesh color related gene and development of molecular marker-assisted selection for flesh color in watermelon (Citrullus lanatus). Front Plant Sci. 2019;10:1240. DOI: 10.3389/fpls.2019.01240.

10. Omoni AO, Aluko RE. The anti-carcinogenic and anti-atherogenic effects of lycopene: a review. Trends Food Sci Technol. 2005;16:344-50. DOI: 10.1016/j.tifs.2005.02.002. 